\title{
Molecular basis of DEL phenotype in the Chinese population
}

\author{
Juan $\mathrm{Gu}^{1,2}$, Xue-Dong Wang ${ }^{1,2^{*}}$, Chao-Peng Shao ${ }^{1,2,3}$, Jun Wang ${ }^{1,2}$, An-Yuan Sun ${ }^{1,2,4}$, Li-Hua Huang ${ }^{1,2}$ \\ and Zhao-Lin Pan ${ }^{1,2}$
}

\begin{abstract}
Background: Rh blood group system is the most complex and immunogenetic blood group system. Prevalent RHD alleles vary in different populations. We conducted the present study to examine the genotype of DEL individuals and to elucidate whether novel alleles exist in the Chinese population.

Methods: DEL phenotype was identified by a serologic adsorption-elution method. The nucleotide sequences of ten RHD exons and exon-intron boundary regions were evaluated by RHD gene-specific PCR-SSP and sequencing.

Results: Of 42306 samples from individual donors and patients, 165 samples were typed as D-negative. Among these D-negative samples, 41 DEL individuals were observed. Thirty-seven DELs were confirmed to have the RHD1227A allele. Two DELs seemed to have RHD-CE-D hybrid alleles, including one RHD-CE (4-7)-D and one RHD-CE (2-5)-D. Two novel RHD alleles were found among the rest of the DEL samples, including one RHD93T > A and one RHD838G > A.

Conclusion: In this study, about 24.85\% (41/165) of the apparent D-negative Chinese individuals were DEL. RHD1227G > A is the most frequent allele in Chinese DEL phenotypes, accounting for 90.24\% (37/41). The RHD-CE-D hybrid allele might be the second most frequent DEL allele in the Chinese population. Our study would contribute to the understanding of the molecular mechanism underlying $D$ antigen expression of DEL individuals and provide useful information for designing suitable genotyping strategies in RhD-negative individuals in Asia.
\end{abstract}

Keywords: Rh blood type, DEL phenotype, RHD allele, Chinese population

\section{Background}

The Rh blood group system is the most complex and immunogenetic blood group system. The Rh antigens are encoded by a pair of homologous genes, $R H D$ and RHCE, which are located on chromosome 1p34.3-36.1. The two genes have opposite orientations at the $R H$ locus, each gene has 10 exons and their sequences are highly homologous. $R H D$ encodes the $\mathrm{D}$ polypeptide, while $R H C E$ gives rise to the $\mathrm{C} / \mathrm{c}$ and E/e polymorphisms. The $R H D$ gene is flanked by an upstream Rh box (at its $5^{\prime}$ end) and a downstream Rh box (at its $3^{\prime}$ end). Both Rh boxes have a length of $\approx 9000$ bp in identical orientation

\footnotetext{
* Correspondence: wxd729@126.com

'Department of Clinical Laboratory, The Fifth People's Hospital of Wuxi, The Clinical College of Nanjing Medical University, Wuxi, Jiangsu 214005, PR

China

${ }^{2}$ The Central Laboratory, Wuxi Fifth Affiliated Hospital of Jiangnan University, Wuxi, Jiangsu 214005, PR China

Full list of author information is available at the end of the article
}

and share $98.6 \%$ homology. The region (breakpoint region) in which the $R H D$ deletion occurs is located within a stretch of $1463 \mathrm{bp}$ in which both Rh boxes have an identical sequence (identity region). The hybrid $\mathrm{Rh}$ box, only present when the $R H D$ gene is deleted via the proposed mechanism of unequal crossing over, contains a sequence identical to that of the upstream $\mathrm{Rh}$ box in the $5^{\prime}$ part preceding the identity region, and the 3 ' part following the identity region is identical to the downstream $\mathrm{Rh}$ box sequence in European people [1]. Studies of the blood group system have shown that racial differences exist not only in the genetic background of the $R H D$ gene but also in the frequencies of the RHD allele [2-5]. About $15 \%$ of European people are D-negative phenotype and are mostly associated with the deletion of RHD between the upstream and the downstream Rhesus boxes. Interestingly, several studies, however, have revealed that there has been the presence of the $R H D$ gene in the majority of 
D-negative Africans and about a quarter of D-negative Africans have an inactive $R H D$ gene of pseudogene $(R H D \psi)$ with a 37 base pair (bp) insertion and a nonsense mutation [6]. In the Asian population, $R H D \psi$ is rare, and a certain percentage of $\mathrm{RhD}$-negative individuals have RHD-CE-D ${ }^{\mathrm{S}}$ hybrid gene or RHD1227A allele. In contrast to the European population, the $\mathrm{RhD}$-negative blood type only occurs in $0.3-0.5 \%$ of the Chinese population; [7] however, nearly $30 \%$ of the $\mathrm{RhD}$-negative individuals belong to the DEL phenotype, which is a rare variant of the Rh system with a grossly intact $R H D$ gene, of which one is the $1227 \mathrm{G}>\mathrm{A}$ mutation that probably disrupts normal intron splicing. In the European population, the frequency of DEL phenotype was 1:3030 and that of RHD1227A allele was 1:9091 [8].

DEL phenotypes derive from several mechanisms. Previous studies showed that DEL phenotype was associated with either a long deletion of $R H D$ gene from intron 8 to intron 9, including whole exon 9, or a missense mutation (RHD1227G > A) in exon 9, or splice-site mutation, including $R H D$ (IVS3 $+1 \mathrm{G}>\mathrm{A}$ ), or a frameshift mutation, including $R H D(\mathrm{X} 418 \mathrm{~L})$ or a mutation in the coding region, or premature stop codons [8-13]. Those studies indicate the D-negative trait may be generated by multiple molecular mechanisms.

DEL is the most frequent $\mathrm{D}$ variant phenotype in the RhD-negative Chinese population that is confirmed by the adsorption-elute test. Moreover, previous studies through genomic DNA analysis showed that the RHD1227 allele is the prevalent causal mutation for DEL individuals in China. Those individuals possess one or two of these alleles with Ccee or CCee phenotypes. Whether there are other DEL alleles in the Chinese population is not yet resolved. The aim of this study was therefore to collect large numbers of blood samples to explore the molecular basis of DEL in the Chinese population.

\section{Methods}

\section{Blood samples}

EDTA-anticoagulated peripheral blood samples were collected from outpatients, inpatient and blood donors at the Anhui Provincial Hospital, the Affiliated Hospital of Anhui Medical University, the Clinical College of Nanjing Medical University, the Affiliated Hospital of Gulin Medical College, the Shaoxing People's Hospital of Zhejiang Province, the $4^{\text {th }}$ Affiliated Hospital of Jiangsu University, Blood Center of Anhui Province, Shenzhen Blood Center of Guangdong Province and Wuxi Blood Center of Jiangsu Province in the southeast China. This study was approved by the institutional Ethics Review Board of Nanjing Medical University and all subjects provided written informed consent to their participation in it.

\section{Serological studies}

Rh blood type phenotype D status was determined by a microplate technique with IgM monoclonal anti-D reagent (Immucor, Norcross, GA; Gamma Biologicals, Houston, TX) [14]. Subsequently, the Rh C/c and E/e antigens were determined by using agglutinating monoclonal anti-C (Novaclone, Dominion Biologicals, Dartmouth, Canada), anti-E (Gamma Biologicals), anti-c (Immucor) and anti-e reagents (Immucor). All samples that were negative with anti-D in the direct agglutination were retested by using the indirect anti-human globulin test (IAT). The indirect anti-human globulin test was used to detect weak D or partial D phenotype. Furthermore, samples typed as D-negative by the IAT were retested for the DEL phenotype through an adsorption-elution test in tubes. If the result was positive $(\geq 1+)$, the sample was determined to belong to the DEL phenotype. If the result was negative, the sample was determined to be truly RhDnegative phenotype.

\section{Molecular studies}

\section{Genomic DNA extraction}

Genomic DNA of DEL samples was prepared using QIAamp DNA Blood Mini Kit according to the manufacturer's recommendations (Qiagen, Valencia, CA, USA). The column-bound circulatory DNA was eluted in distilled water and quantified by using a spectrophotometer (NanoDrop 2000c, Thermo Scientific, Wilmington, DE, USA).

\section{Amplification of $R H D$ exons}

Primers for $R H D$ exons 1 to 10 were given in Table 1 . $\beta$ actin gene was used as an internal control. Amplification was carried out in a thermal cycler (Veriti 96, Applied Biosystems, Foster City, CA). Cycling conditions for $R H D$ exon 1 and 10 were denatured at $95^{\circ} \mathrm{C}$ for $5 \mathrm{~min}$, then 40 cycles of $30 \mathrm{~s}$ at $94^{\circ} \mathrm{C}$, annealing time was $40 \mathrm{sec}-$ onds at $64^{\circ} \mathrm{C}$ (exon 2-4, 6, 8), $62^{\circ} \mathrm{C}$ (exon 1, 7, 9, 10, RHD1227A) and $60^{\circ} \mathrm{C}$ (exon 5), and extension for $1 \mathrm{~min}$ at $72^{\circ} \mathrm{C}$. The final extension was at $72^{\circ} \mathrm{C}$ for 10 minutes. PCR products were visualized by electrophoresis on a 1.5\% agarose gel with ethidium bromide staining and these were photographed under UV light.

\section{PCR-restriction fragment length polymorphism (PCR-RFLP) for RHD zygosity determination}

The PCR amplification was performed by using the expand high-fidelity PCR system with primers rez7 (consensus, $5^{\prime}$ of the $\mathrm{Rh}$ box identity region) and rnb31 (specific for downstream of the Rh box, 3' of the Rh box identity region) [1]. PCR products were digested with Pst I at $37^{\circ} \mathrm{C}$ for $3 \mathrm{~h}$, and the fragments were resolved by electrophoresis on a $1.5 \%$ agarose gel with ethidium 
Table 1 Primers used for PCR analysis and DNA sequencing

\begin{tabular}{|c|c|c|c|c|}
\hline Primer name* & Sequence $5^{\prime}$ to $3^{\prime}$ & Location $^{\#}$ & Specificity & Product size (bp) \\
\hline E1-s & ATGCCTGGTGCTGGTGGA & promoter, -43 to -26 & RHD/CE & \multirow{2}{*}{228} \\
\hline E1-a & ATTTGCTCCTGTGACCACTT & \multirow[t]{2}{*}{ intron 1,37 to 18} & \multirow[t]{2}{*}{ RHD } & \\
\hline E1-seq & ATGCCTGGTGCTGGTGGA & & & \\
\hline E2-s & TGACGAGTGAAACTCTATCTCGAT & intron $1,-1064$ to -1041 & $R H D$ & \multirow{3}{*}{1475} \\
\hline E2-a & GGATTCCTTGTGATACACGGAGTAG & \multirow[t]{2}{*}{ intron 2, 224 to 200} & \multirow[t]{2}{*}{ RHD } & \\
\hline E2-seq & TGACGAGTGAAACTCTATCTCGAT & & & \\
\hline E3-s & GTCGTCCTGGCTCTCCCTCTCT & intron $2,-29$ to -8 & $R H D$ & \multirow{3}{*}{219} \\
\hline E3-a & CTITTCTCCCAGGTCCCTCCT & \multirow[t]{2}{*}{ intron 3,39 to 19} & \multirow[t]{2}{*}{ RHD/CE } & \\
\hline E3-seq & GTCGTCCTGGCTCTCCCTCTCT & & & \\
\hline E4-s & GCCGACACTCACTGCTCTTAC & intron $3,-36$ to -16 & $R H D / C E$ & \multirow{3}{*}{378} \\
\hline E4-a & TGAACCTGCTCTGTGAAGTGC & \multirow[t]{2}{*}{ intron 4,194 to 174} & \multirow[t]{2}{*}{ RHD } & \\
\hline E4-seq & GCCGACACTCACTGCTCTTAC & & & \\
\hline E5-s & TACCTTTGAATTAAGCACTTCACAG & intron $4,-267$ to -233 & $R H D$ & \multirow{3}{*}{1458} \\
\hline E5-a & TTATTGGCTACTTGGTGCC & \multirow[t]{2}{*}{ intron 5, 1024 to 1006} & \multirow[t]{2}{*}{$R H D$} & \\
\hline E5-seq & TACCTTGAATTAAGCACTTCACAG & & & \\
\hline E6-s & CAGGGTTGCCTTGTTCCCA & intron $5,-95$ to -77 & RHD/CE & \multirow{3}{*}{274} \\
\hline E6-a & CTTCAGCCAAAGCAGAGGAGG & \multirow[t]{2}{*}{ intron 6,41 to 21} & \multirow[t]{2}{*}{ RHD } & \\
\hline E6-seq & CAGGGTTGCCTTGTTCCCA & & & \\
\hline E7-s & TGCCCATCCCCCTITGGTGGCC & intron $6,-106$ to -85 & $R H D$ & \multirow{3}{*}{411} \\
\hline E7-a & CCAAGGTAGGGGCTGGACAG & \multirow[t]{2}{*}{ intron 7,171 to 152} & \multirow[t]{2}{*}{ RHD } & \\
\hline E7-seq & TGCCCATCCCCCTITGGTGGCC & & & \\
\hline E8-s & GGTCAGGAGTTCGAGATCAC & intron $7,-594$ to -575 & $R H D$ & \multirow{3}{*}{709} \\
\hline E8-a & TGGCAATGGTGGAAGAAAG & \multirow[t]{2}{*}{ intron 8,35 to 16} & \multirow[t]{2}{*}{ RHD/CE } & \\
\hline E8-seq & GGTCAGGAGTTCGAGATCAC & & & \\
\hline E9-s & CTGTCGTTITGACACACAATATTTC & intron $8,-91$ to -67 & $R H D$ & \multirow{3}{*}{190} \\
\hline E9-a & CACGTTAATAGGTGAAAAATCTTACC & \multirow[t]{2}{*}{ intron 9,25 to exon 9, 1227} & \multirow[t]{2}{*}{$R H D$} & \\
\hline E9-seq & CTGTCGTTITGACACACAATATTTC & & & \\
\hline E10-s & CAAGAGATCAAGCCAAAATCAGT & intron $9,-67$ to -45 & $R H D / C E$ & 307 \\
\hline E10-a & AGCTTACTGGATGACCACCA & \multirow[t]{2}{*}{ 3'UTR, 291 to 272} & \multirow[t]{2}{*}{ RHD } & 502 \\
\hline E10-seq & CAAGAGATCAAGCCAAAATCAGT & & & \\
\hline RHD1227A-s & GATGACCAAGTITTCTGGAAA & exon 9, 1207 to 1227 & RHD1227A & 100 \\
\hline RHD1227A-a & CATAAACAGCAAGTCAACATATATACT & intron9, 88 to 62 & $R H D$ & 109 \\
\hline RHD1227A-seq & GATGACCAAGTITCTGGAAA & & & \\
\hline$\beta$-actin-s & GGAAATCGTGCGTGACATT & - & & 473 \\
\hline$\beta$-actin-a & CGTCATACTCCTGCTTGCTG & - & & \\
\hline
\end{tabular}

${ }^{*} \mathrm{~s}=$ sense primer; $\mathrm{a}=$ antisense primer. seq $=$ sequencing primer.

"The positions of the synthetic oligonucleotides are indicated relative to their distances from the first nucleotide position of the start codon ATG for all primers in the promoter and in the exons or relative to their adjacent exon-intron boundaries for all other primers.

bromide staining and these were photographed under UV light.

\section{Purification and sequencing}

Amplified DNA products were purified using an isolation kit (NucleoSpin Extract II, Macherey-Nagel, Düren, Germany) and sequenced on a genetic analyzer by DNA technology (ABI 3130 XL, Applied Biosystems, Aarhus,
Denmark). The complete $R H D$ exons 1 to 10 including adjacent intron regions were sequenced from PCR products with cycle-sequencing kits (BigDye-terminators v.1.1; Applied Biosystems, Weiterstadt, Germany) and Sequence determination of amplicons was performed on both strands using the respective PCR primers with an ABI PRISM 3730 automated sequencer (Applied Biosystems, Foster City, USA). The nucleotide and deduced 
amino acid sequences were analyzed and compared with the published sequences using computer software (Lasergene 99, DNASTAR, Madison, WI) as a sequencing analysis tool. All sequenced PCR products were compared with GenBank Accession Number BN000065.

\section{Results}

Serologic studies

A total of 165 apparent D-negative samples were found among the 42306 blood donors and patients through the microplate determination. When the D-negative samples were retested by the IAT and the adsorption-elution test, a total of 41 DELs were found among these samples. In the Chinese population examined, approximately $24.58 \%$ $(41 / 165)$ of the apparent D-negative individuals belong to DEL phenotypes (Table 2).

\section{Molecular characterization of DELs}

Among the 41 DELs samples, a total of 37 samples were determined to have the RHD1227A allele by PCR-SSP analysis and sequencing (Table 2, Figures $1 \mathrm{a}$ and 2a), accounting for $90.24 \%$ (37/41) in the Chinese DEL individuals. All of these individuals carrying RHD1227A allele were demonstrated that the mutation is located in the splice site at the exon 9 and intron 9 junctions. The remaining four DEL samples did not have RHD1227A (Figure 1a; lines 6 to 9). Two samples seemed to have RHD-CE-D hybrid alleles. According to the PCR-SSP for the $R H D$ gene exons 1 to 10 determination, the first sample (sample No: 13039 ) lacked $R H D$ exons 4 to7, the second sample (sample No: 13038) lacked exons 2 to 5 (Figure $1 \mathrm{~b}$ and $\mathrm{c}$ ). The two samples were confirmed to be $R H D-C E$ (4-7)-D and $R H D-C E$ (2-5)-D, respectively. No mutation was found in the $R H D$-specific exons by sequencing.

Two novel $R H D$ alleles were found in the remaining two DEL samples. One sample (sample No: 13040) was found to have a $R H D 93 \mathrm{~T}>\mathrm{A}$ mutation (Figure 2b), the other sample (sample No: 13041) had a RHD838G > A mutation (Figure 2c). Their DNA sequences were deposited under GenBank accession numbers: RHD93T $>\mathrm{A}$ (KJ558352 for exon 1), RHD838G > A (KJ558353 for exon 6). The RHD93T $>\mathrm{A}$ and RHD838G $>\mathrm{A}$ alleles were missense mutations, which caused F31L and A280T amino acid mutations, respectively, both of the two mutations occurred in the $\mathrm{RhD}$ transmembrane domain. The samples with $R H D 93 \mathrm{~T}>\mathrm{A}$ and $R H D 838 \mathrm{G}>\mathrm{A}$ alleles belong to Ccee phenotype. The sample assigned to RHD93T > A allele was found to be $R H D+/ R H D+$ homozygote by PCR-RFLP analysis and the other sample with $R H D 838 \mathrm{G}>\mathrm{A}$ allele was $R H D+/ R H D$ - heterozygote (Table 2).

\section{Discussion}

DEL phenotype was determined on the basis of nonagglutination by the IAT procedure and positive result by an adsorption-elution technique. Molecular studies have shown that a heterogeneous array of variant $R H D$ allele can result in the DEL phenotype [15]. Körmöczi and colleagues suggested that the DEL phenotypes might be subdivided into two groups, partial DEL with characteristic D epitope loss caused by either $R H D-C E-\mathrm{D}$ hybrid genes or $R H D$ point mutation such as carrier of RHD (IVS3 + 1G > A) affecting extracellular RhD loops and complete DEL where the majority of D epitopes are conserved such as RHD1227A [16]. According to the published data [9,17] and our results, the RHD1227A allele was the most frequent allele in the Chinese DEL individuals. In our study, two DELs seemed to have RHD$C E$-D hybrid alleles, including one RHD-CE (4-7)-D and one $R H D-C E$ (2-5)-D. The RHD-CE (4-7)-D was thought to be a DEL allele by our laboratory, while a similar hybrid RHD-CE (4-7)-D was described as D-negative [10]. Whether there were miniscule differences between these two alleles has not yet been clarified. The $R H D-C E$ (2-5)-D was similar to the $R H$ allele that is also called $\mathrm{D}^{\mathrm{VI}}$

Table 2 Results of phenotype and genotype analyses of the 41 DEL samples

\begin{tabular}{|c|c|c|c|c|c|c|c|}
\hline \multirow{2}{*}{ Sample no. } & \multirow{2}{*}{$\begin{array}{l}\text { Number } \\
\text { of subjects }\end{array}$} & \multicolumn{3}{|c|}{ RhD } & \multirow{2}{*}{ RhCcEe } & \multirow{2}{*}{$\begin{array}{c}R H D \\
\text { zygosity* }\end{array}$} & \multirow{2}{*}{$R H D$ allele } \\
\hline & & Micro-plate & IAT & Adsorption/elute & & & \\
\hline 13001-13030 & 30 & - & - & + & Ccee & $R H D+/ R H D-$ & RHD1227A \\
\hline 13031-13033 & 3 & - & - & + & CCee & $R H D+/ R H D-$ & RHD1227A \\
\hline 13034-13035 & 2 & - & - & + & Ccee & $R H D+/ R H D+$ & RHD1227A \\
\hline 13036 & 1 & - & - & + & CCEe & $R H D+/ R H D-$ & RHD1227A \\
\hline 13037 & 1 & - & - & + & CcEe & $R H D+/ R H D-$ & RHD1227A \\
\hline 13038 & 1 & - & - & + & Ccee & $R H D+/ R H D-$ & RHD-CE (2-5)-D \\
\hline 13039 & 1 & - & - & + & Ccee & $R H D+/ R H D-$ & RHD-CE (4-7)-D \\
\hline 13040 & 1 & - & - & + & Ccee & $R H D+/ R H D+$ & RHD93T $>A$ \\
\hline 13041 & 1 & - & - & + & Ccee & $R H D+/ R H D-$ & $R H D 838 G>A$ \\
\hline
\end{tabular}

*Presence (+) or absence (-) of the RHD gene. 

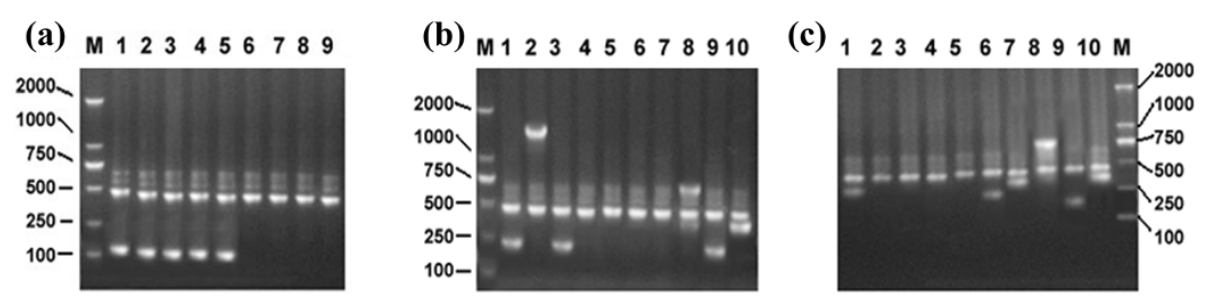

Figure 1 Results of the PCR-SSP analysis. In all lanes, a 473-bp product was amplified as the internal positive control; M, molecular marker (2000, 1000, 750, 500, 250 and 100 bp, respectively). (a) PCR-SSP for RHD1227A allele in 9 DEL samples. Lanes 1 to 9 showed the PCR-SSP results of 9 DEL samples. Lanes 1 to 5 showed that the RHD1227A specific amplifications (band of 109 bp) were positive (Sample No.13001-13005). They were shown as representatives of 37 DEL individuals carrying RHD1227A allele. Lane 6 to 9 showed that the RHD1227A specific amplifications were negative (Sample No.13038-13041). (b) PCR-SSP for RHD-CE (4-7)-D in one DEL sample (Sample No.13039). Lanes 1 to 10 showed the PCR-SSP results of the RHD exons 1 to 10; Lanes 4 to 7 showed that the RHD specific amplifications were negative for exons 4 to 7; RHD specific amplifications were positive for exons 1 to 3 and 8 to 10. (c) PCR-SSP for RHD-CE (2-5)-D in one DEL sample (Sample No.13038); lanes 1 to 10 showed the PCR-SSP results of the RHD exons 1 to 10; Lanes 2 to 5 showed that the RHD specific amplifications were negative for exons 2 to 5 ; RHD specific amplifications were positive for exons 1, 6 to 10.

type 4. Hasekura et al. have suggested that some DELs were caused by a trans effect of $C$ on a haplotype containing a weak D allele [18]. Whether the $\mathrm{D}^{\mathrm{VI}}$-like DEL has a similar mechanism, that the DEL was resulted from the influence of $\mathrm{C}$ in trans on a haplotype with a partial $\mathrm{D}$ allele, needs to be further analysed. The hybrid gene individuals were detected with $\mathrm{Rh}$ box zygosity. They were found to be $R H D+/ R H D$ - heterozygote (Table 2). These results may also be in concordance with the studies of Li et al [17].
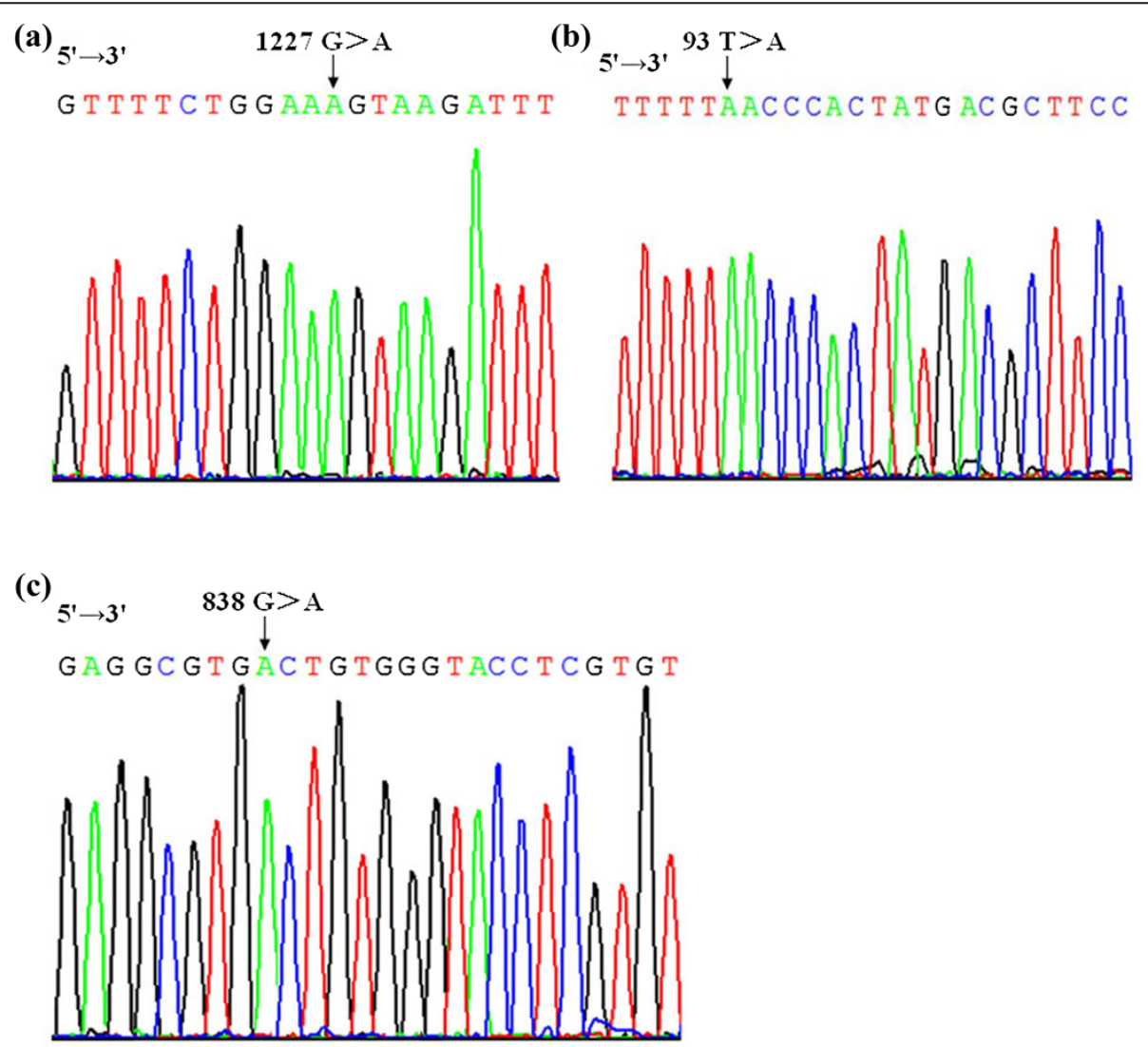

Figure 2 Electropherograms of RHD DNA sequencing. (a) Sequencing analysis of the RHD1227A allele. The arrow indicates the position of nucleotide mutation between RHD exon 9 and intron 9. A representative example of 37 RHD1227A genotyped cases is shown; (b) Sequencing analysis of the RHD93T > A allele in one DEL sample (Sample No.13040). The arrow indicates the position of nucleotide mutation in RHD exon 1; (c) Sequencing analysis of the RHD838G > A allele in one DEL sample (Sample No.13041). The arrow indicates the position of nucleotide mutation in $R H D$ exon 6. 
Two samples with the novel $R H D 93 \mathrm{~T}>\mathrm{A}$ and RHD838G > A alleles showed slightly stronger positive results $(2+)$ than other DEL samples $(1+)$ in the adsorptionelution test. This phenomenon suggested that there might be scant differences in the $\mathrm{D}$ antigen between these two samples and other DELs. The possibility that these two samples might be two new kinds of weak $D^{S}$ could not be excluded.

DEL is the weakest D positive phenotype, whether the potential danger that DEL RBCs might cause a clinical transfusion reaction cannot be completely excluded. Recipients with a truly D-negative phenotype developed anti-D after transfusion with DEL RBCs $[19,20]$. Recently, Richard and his colleagues found a patient with a DEL phenotype who developed anti-D [11,12]. Other members of our team have not yet reported analogous cases in the Chinese population. Shao and his colleagues found that the Asian type DEL displays the complete repertoire of $\mathrm{RhD}$ antigen epitopes. They suggested that people in East Asia who carry the DEL variants could safely receive blood transfusion from RhDpositive donors [21]. Our previous study also supported the biochemical observations that DEL variants express normal $\mathrm{RhD}$ and pose virtually no risk of inducing anti-D antibodies [22]. We also confirmed that this is an exact model test that people carrying the DEL variants can safely receive blood transfusion from $\mathrm{RhD}$-positive donors.

Throughout the world, the majority of DEL phenotypes are misinterpreted as D-negative owing to the limits of routine typing. For this reason, the recipient with a truly D-negative phenotype would be likely to develop into anti-D alloimmunization after transfusion with DEL RBCs. So it would be of interest to routinely screen serologically D-negative donors for the presence of the $R H D$ gene in order to discriminate all clinically relevant RHD DEL alleles. An optimized PCR strategy that checks for $R H D$-specific polymorphism, supplemented by the specific detection of aberrant alleles, would be a good choice. Samples carrying $R H D$ deletion should be correctly typed by multiple PCR utilized for $R H D$ genotyping. Testing additional $R H D$ exons would improve the specificity of RhD prediction. Although direct sequencing is currently the gold standard in mutation identification, it is still relatively laborious and expensive, whereas PCR-SSP as a onestep method is less expensive and less time consuming. In any case, the combined use of serologic and molecular D typing techniques may reduce the number of such transfusion incidents. All of these findings suggested that it might be important to explore the molecular basis of DEL individuals in Asia and to form molecular screening techniques to determine the DEL phenotype accurately.

\section{Conclusion}

In this study, about $24.85 \%(41 / 165)$ of the apparent D-negative Chinese individuals were DEL. RHD1227G > A is the most frequent allele in Chinese DEL phenotypes, accounting for $90.24 \%$ (37/41). The RHD-CE-D hybrid allele might be the second most frequent DEL allele in the Chinese population. Novel DEL alleles are still relatively rare and frequencies of occurrence are also very low. Our study would contribute to the understanding of the molecular mechanism underlying D antigen expression of DEL individuals and provide useful information for designing suitable genotyping and transfusion strategies for the RhD-negative individuals in Asia.

\section{Competing interests}

The authors declare that they have no competing interests.

\section{Authors' contributions}

Authors who obtained funding included XDW and JG; Authors who participated in conception and design were XDW, JG and CPS; Authors who carried out data analysis and interpretation included JG, AYS, JW, ZLP and $\mathrm{LHH}$; Authors who drafted and finalized the manuscript were XDW, JG, and CPS. All authors read and approved the final manuscript.

\section{Acknowledgements}

This work was supported by the Medical Research Foundation of Wuxi Municipal Health Bureau (Grant No. ML201203) and the Scientific Technology Development Foundation of Nanjing Medical University (Grant No. 2012NJMU071). The funders had no role in the study design, data collection or analysis, decision to publish, or preparation of the manuscript.

\section{Author details}

'Department of Clinical Laboratory, The Fifth People's Hospital of Wuxi, The Clinical College of Nanjing Medical University, Wuxi, Jiangsu 214005, PR China. ${ }^{2}$ The Central Laboratory, Wuxi Fifth Affiliated Hospital of Jiangnan University, Wuxi, Jiangsu 214005, PR China. ${ }^{3}$ Department of Transfusion, The Second People's Hospital of Shenzhen, Guangdong 518035, PR China. ${ }^{4}$ Department of Clinical Laboratory, The Affiliated Provincial Hospital of Anhui Medical University, Hefei, Anhui 230031, PR China.

Received: 28 November 2013 Accepted: 30 April 2014 Published: 5 May 2014

\section{References}

1. Wagner FF, Flegel WA: RHD gene deletion occurred in the Rhesus box. Blood 2000, 95:3662-3668.

2. Pham BN, Roussel M, Peyrard T, Beolet M, Jan-Lasserre V, Gien D, Ripaux M, Bourgouin S, Kappler-Gratias S, Rouger P, Le Pennec PY: Anti-D investigations in individuals expressing weak D Type 1 or weak D Type 2: allo- or autoantibodies? Transfusion 2011, 51:2679-2685.

3. Lin IL, Shih MC, Hsieh MH, Liu TC, Chang SE, Lin CL, Chang JG: Molecular basis of weak D in Taiwanese. Ann Hematol 2003, 82:617-620.

4. Silvy M, Chapel-Fernandes S, Callebaut I, Beley S, Durousseau C, Simon S, Lauroua P, Dubosc-Marchenay N, Babault C, Mouchet C, Ferrera V, Chiaroni J, Bailly P: Characterization of novel RHD alleles: relationship between phenotype, genotype, and trimeric architecture. Transfusion 2012, 52:2020-2029.

5. Brajovich ME, Boggione CT, Biondi CS, Racca AL, Tarragó M, Nogués N, Muñiz-Díaz E, Cotorruelo CM: Comprehensive analysis of $R H D$ alleles in Argentineans with variant D phenotypes. Transfusion 2012, 52:389-396.

6. Singleton BK, Green CA, Avent ND, Martin PG, Smart E, Daka A, Narter-Olaga EG, Hawthorne LM, Daniels G: The presence of an RHD pseudogene containing a $37 \mathrm{bp}$ duplication and a nonsense mutation in Africans with the Rh D-negative blood group phenotype. Blood 2000, 95:12-18.

7. Peng CT, Shih MC, Liu TC, Lin IL, Jaung SJ, Chang JG: Molecular basis for the RhD negative phenotype in Chinese. Int J Mol Med 2003, 11:515-521. 
8. Wagner FF, Frohmajer A, Flegel WA: RHD positive haplotypes in D negative Europeans. BMC Genet 2001, 2:10.

9. Shao CP, Maas JH, Su YQ, Köhler M, Legler TJ: Molecular background of Rh D- positive, D-negative, D (el) and weak D phenotypes in Chinese. Vox Sang 2002, 83:156-161.

10. Gassner C, Doesher A, Drnovsek D, Rozman P, Eicher NI, Legler TJ, Lukin S, Garritsen H, Kleinrath T, Egger B, Ehling R, Körmöczi GF, Kilga-Nogler S, Schoenitzer D, Petershofen EK: Presence of RHD in serologically D-, C/E + individuals: a European multicenter study. Transfusion 2005, 45:527-538.

11. Richard M, Perreault J, Constanzo-Yanez J, Khalifé S, St-Louis M: A new DEL variant caused by exon 8 deletion. Transfusion 2007, 47:852-857.

12. Luettringhaus TA, Cho D, Ryang DW, Flegel WA: An easy RHD genotyping strategy for D- East Asian persons applied to Korean blood donors. Transfusion 2006, 46:2128-2137.

13. Xu Q, Grootkerk-Tax MG, Maaskant-van Wijk PA, van der Schoot CE: Systemic analysis and zygosity determination of the RHD gene in a D-negative Chinese Han population reveals a novel D-negative RHD gene. Vox Sanguinis 2005, 88:35-40.

14. Brecher M: Red cell typing methods. In Technical Manual. 14th edition. Edited by Brecher M. American Association of Blood Banks: Bethesda, MD; 2002:677-679.

15. Gardener GJ, Legler TJ, Hyett JA, Liew YW, Flower RL, Hyland CA: Anti-D in pregnant women with the RHD (IVS3 + 1G > A)-associated DEL phenotype. Transfusion 2012, 52:2016-2019.

16. Körmöczi GF, Gassner C, Shao CP, Uchikawa M, Legler TJ: A comprehensive analysis of DEL types: partial DEL individuals are prone to anti-D alloimmunization. Transfusion 2005, 45:1561-1567.

17. Li Q, Hou L, Guo ZH, Ye LY, Yue DQ, Zhu ZY: Molecular basis of the RHD gene in blood donors with DEL phenotypes in Shanghai. Vox Sanguinis 2009, 97:139-146.

18. Hasekura H, Ota M, Ito $\mathrm{S}$, Hasegawa $\mathrm{Y}$, Ichinose A, Fukushima H, Ogata H: Flow cytometric studies of the $D$ antigen of various Rh phenotypes with particular reference to Du and Del. Transfusion 1990, 30:236-238.

19. Wagner T, Körmöczi GF, Buchta C, Vadon M, Lanzer G, Mayr WR, Legler TJ: Anti-D immunization by DEL red blood cells. Transfusion 2005, 45:520-526.

20. Yasuda $\mathrm{H}$, Ohto $\mathrm{H}$, Sakuma S, Ishikawa Y: Secondary anti-D immunization by Del red blood cells. Transfusion 2005, 45:1581-1584.

21. Shao CP: Transfusion of RhD-Positive Blood in "Asia Type" DEL Recipients. N Engl J Med 2010, 362:472-473.

22. Wang QP, Dong GT, Wang XD, Gu J, Li Z, Sun AY, Shao CP, Pan ZL, Huang $L H$, Xie WX, Sun GM, Chen JJ, Pei H, Yang XJ, Shan PN: An investigation of secondary anti-D immunisation among phenotypically RhD-negative individuals in the Chinese population. Blood Transfus 2013, 1-6. doi:10.2450/2013.0184-12.

\section{Submit your next manuscript to BioMed Central and take full advantage of:}

- Convenient online submission

- Thorough peer review

- No space constraints or color figure charges

- Immediate publication on acceptance

- Inclusion in PubMed, CAS, Scopus and Google Scholar

- Research which is freely available for redistribution

Submit your manuscript at www.biomedcentral.com/submit
C Biomed Central 